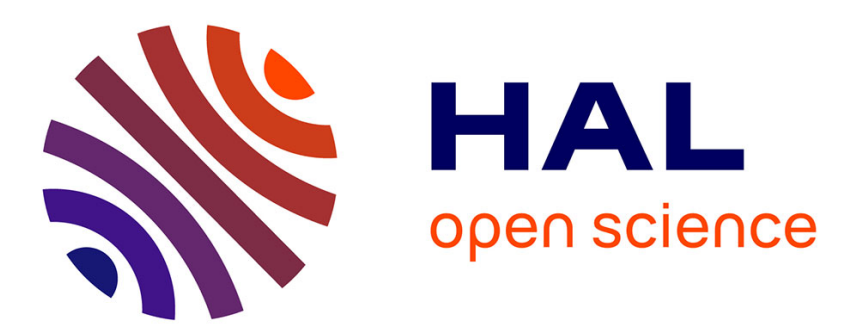

\title{
Activity Pattern Impact of Primary Radio Nodes on Channel Selection Strategies
}

Mubashir Husain Rehmani, Aline Carneiro Viana, Hicham Khalife, Serge Fdida

\section{- To cite this version:}

Mubashir Husain Rehmani, Aline Carneiro Viana, Hicham Khalife, Serge Fdida. Activity Pattern Impact of Primary Radio Nodes on Channel Selection Strategies. CogART 2011 - 4th International Workshop on Cognitive Radio and Advanced Spectrum Management, Oct 2011, Barcelona, Spain. pp.36:1-36:5, 10.1145/2093256.2093292 . inria-00630230

\section{HAL Id: inria-00630230 \\ https://hal.inria.fr/inria-00630230}

Submitted on 7 Oct 2011

HAL is a multi-disciplinary open access archive for the deposit and dissemination of scientific research documents, whether they are published or not. The documents may come from teaching and research institutions in France or abroad, or from public or private research centers.
L'archive ouverte pluridisciplinaire HAL, est destinée au dépôt et à la diffusion de documents scientifiques de niveau recherche, publiés ou non, émanant des établissements d'enseignement et de recherche français ou étrangers, des laboratoires publics ou privés. 


\title{
Activity Pattern Impact of Primary Radio Nodes on Channel Selection Strategies
}

\author{
Mubashir Husain Rehmani ${ }^{\star}$, Aline Carneiro Viana ${ }^{\ddagger}$, Hicham Khalife $^{\dagger}$, and Serge Fdida ${ }^{\star}$ \\ * LIP6/UPMC Sorbonne Universités $\quad \ddagger$ INRIA $\quad$ LaBRI/ENSEIRB, Université de Bordeaux \\ mubashir.rehmani@lip6.fr, aline.viana@inria.fr, hicham.khalife@labri.fr, serge.fdida@lip6.fr
}

\begin{abstract}
The performance of cognitive radio network is highly dependent upon the primary radio nodes activity pattern. In this paper, we study and analyze the impact of different PR nodes activity pattern with the help of three performance metrics. In this perspective, we use our channel selection strategy SURF and three other channel selection strategies i.e., Random (RD), Highest Degree (HD), and Selective Broadcasting (SB). We analyze the performance of these channel selection strategies through extensive NS-2 simulations. Moreover, we also analyze how these strategies respond to different PR nodes activity. Simulation results confirm that SURF outperforms RD, HD, and SB in terms of delivery ratio and causes less harmful interference to PR nodes, in all primary radio nodes activity pattern.
\end{abstract}

\section{Categories and Subject Descriptors}

C.2.1 [Computer-Communication Networks]: Network Architecture and Design-Wireless communication

\section{General Terms}

Wireless Communications

\section{Keywords}

Multi-hop cognitive radio networks, channel selection, data dissemination, primary radio activity.

\section{INTRODUCTION}

Cognitive radio wireless networks (CRNs) [1] are designed to use the radio spectrum opportunistically. CRNs are composed of two types of nodes: Primary Radio (PR) nodes and the Cognitive Radio (CR) nodes. Primary radio nodes are the legacy users and they have the high priority to use the channels for communication. However, cognitive radio nodes can only use the channels when they are idle i.e. not utilized by the PR nodes. Therefore, the performance of cognitive radio network is highly dependent upon the primary radio nodes activity pattern. The primary radio nodes activity pattern i.e. presence or absence of the PR signal, can be modelled as continuous-time, alternating ON/OFF Markov Renewal Process (MRP) $[2,3,4]$. This PR activity model has been used very widely in the literature $[2,3,4]$.

Recently, very few works has been done to analyze PR nodes activity pattern. In [5], the authors model and evaluate the performance of Transmission Control Protocol over Cognitive Radio Ad Hoc Networks. The authors considered a single-hop topology for PR activity analysis and four different regions (long term, high, low, intermittent) for PR nodes activity. The effect of PR ON/OFF periods on the system performance in the context of MAC protocol has been evaluated by Bayhan and Alagöz [6]. In [7], the authors studied the influence of the activity patterns of the primary radio transmitters on the area in which cognitive radios have opportunities for spectrum reuse, with the given transmit power. But none of these works have analyzed the impact of different $\mathrm{PR}$ nodes activity pattern on different channel selection strategies as well as on data dissemination. Moreover, these works do not consider the effect of PR nodes activity in a multi-hop network. In fact, due to lack of centralized entity and the difficult coordination between $\mathrm{CR}$ nodes in multi-hop cognitive radio ad-hoc network, the selection of a common channel by CR transmitters and receivers is a challenging task.

In this paper, we study and analyze the impact of different PR nodes activity patterns on different channel selection strategies i.e. Random (RD), Highest Degree (HD), Selective Broadcasting (SB) and our proposed channel selection strategy (SURF). Moreover, we also analyzed how these channel selection strategies respond to different PR activity patterns. In particular, by analyzing our channel selection strategy SURF [8] under different PR activity patterns (wireless environments), we gain insights that will help us in future to set up different channel heuristics. Through extensive NS-2 simulations, we generate different PR activity patterns and investigate through several performance parameters how the approaches react.

The remainder of this paper is organized as follows: Section 2 give a brief over of channel selection strategies i.e. RD, HD, SB, and SURF. In section 3, we discuss the PR nodes activity patterns. Performance evaluation is done in section 4, improvements regarding SURF are suggested in section 5 and finally, section 6 concludes the paper.

\section{CHANNEL SELECTION STRATEGIES}

We consider four channel selection strategies i.e. Random (RD), Highest Degree (HD), Selective Broadcasting (SB), and our proposed channel selection strategy (SURF). We now describe each of them.

In $\mathrm{RD}$ approach, channels are randomly selected to be used by CR nodes for transmission and/or overhearing, without any consideration to the ongoing PR and CR activity over these channels.

In SB [9], each CR node calculates a minimum set of channels, Essential Channel Set (ECS), for transmission that covers all its geographic neighbors, without considering the PR unoccupancy. In SB, a CR node transmits on multiple chan- 
nels in round-robin fashion present in the ECS list, until all neighbors are covered. Note that in [9] nothing is mentioned about how nodes overhear over the channels. Therefore, we consider nodes select for overhearing the highest degree channel from their ECS list only. If more than one option is available, a random choice for transmission/overhearing is performed among those channels with the same degree.

HD approach only considers $\mathrm{CR}$ activities and is inspired by $\mathrm{SB}$ approach. In $\mathrm{HD}, \mathrm{CR}$ nodes select the highest $\mathrm{CR}$ degree channel for transmission and overhearing, without any consideration of PR activity. The highest degree channel covers, consequently, the highest number of neighbors in the available list of channels.

SURF [8] is our distributed channel selection strategy specifically designed for data dissemination in multi-hop cognitive radio networks. In SURF, special consideration is given to select those channels that cause less harmful interference to PR nodes. This is achieved by considering the PR nodes activity pattern during the channel selection decision. On top of that, SURF gives high preference to those channels that have higher number of CR neighbors.

SURF strategy classifies channels by assigning a weight $P_{w}^{(i)}$ to each observed channel $i$ in the channel set $C$. Thus, every cognitive radio running SURF, locally computes the $P_{w}^{(i)}$ using the following equation:

$$
\forall i \in C: P_{w}^{(i)}=P R_{u}^{(i)} \times C R_{o}^{(i)}
$$

$P_{w}^{(i)}$ describes the weight of a channel $i$ and is calculated based on the unoccupancy of PR (i.e. $P R_{u}^{(i)}$ ) and CR occupancy (i.e. $C R_{o}^{(i)}$, which reflects the number of CR neighbors) over channel $i$. Then, the channels are ranked according to their weights and the best channel (i.e., the one providing highest $P_{w}^{(i)}$ ) is selected. SURF has also the mechanism of recovery from bad channel selection decision, which is considered during the computation of the primary radio unoccupancy. In this mechanism, SURF keeps track of previous wrong channel state estimation and accordingly adapts future channel selection decision. The primary radio unoccupancy $P R_{u}^{(i)}$ is given by:

$$
P R_{u}^{(i)}=P_{O F F}^{*}(t)^{(i)}=P_{O F F}^{(i)}\left(1-P_{F A}^{(i)}\right)+P_{M D}^{(i)}\left(1-P_{O F F}^{(i)}\right)
$$

where $P_{O F F}(t)$ (cf. Eq. 3) is the probability that the channel $i$ will be in OFF state at time $t, P_{F A}^{(i)}$ is the probability of false alarm, and $P_{M D}^{(i)}$ is the probability of miss-detection. More details on how these probabilities are calculated can be found in our paper [8] and technical report [10].

\section{PR NODES ACTIVITY PATTERN}

The primary radio nodes activity, i.e. presence or absence of the PR signal, can be modelled as continuous-time, alternating ON/OFF Markov Renewal Process (MRP) [2, 3, $4,15]$. This PR activity model has been used very widely in the literature $[2,3,4]$. The ON/OFF PR activity model approximates the spectrum usage pattern of public safety bands [11]. The public safety band is designated for commercial and public safety uses [12]. The authors in [13] approximate and validate the $\mathrm{PR} \mathrm{ON} / \mathrm{OFF}$ activity model for the presence of the PR signal in IEEE 802.11b. The ON/OFF PR activity model is also the most famous model for voice [14].

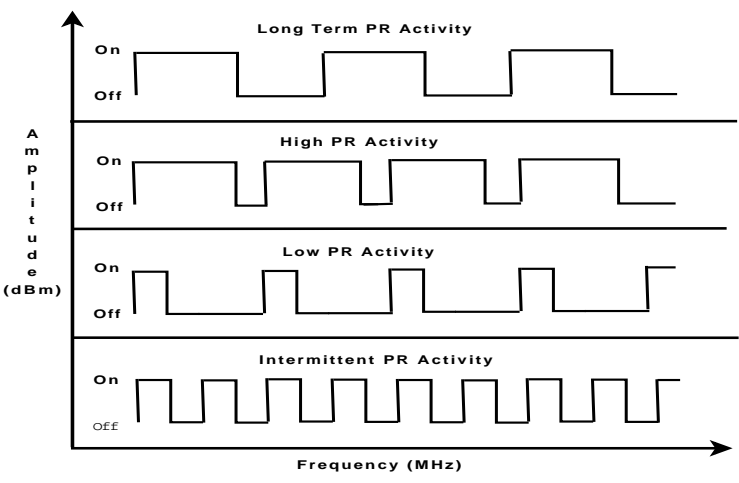

Figure 1: Long term, high, low and intermittent PR nodes activity.

Table 1: Primary Radio Activity.

\begin{tabular}{|c|c|c|c|c|}
\hline PR Activity & ON & OFF & $\lambda_{X}$ & $\lambda_{Y}$ \\
\hline \hline Long Term Activity & $\lambda_{X} \leq 1$ & $\lambda_{Y} \leq 1$ & Long ON & Long OFF \\
\hline High Activity & $\lambda_{X} \leq 1$ & $\lambda_{Y}>1$ & Long ON & Short OFF \\
\hline Low Activity & $\lambda_{X}>1$ & $\lambda_{Y} \leq 1$ & Short ON & Long OFF \\
\hline Intermittent Activity & $\lambda_{X}>1$ & $\lambda_{Y}>1$ & Short ON & Short OFF \\
\hline
\end{tabular}

In this paper, we use the formulation of $[2,4,15]$ that the channels ON and OFF periods are both exponentially distributed with p.d.f. $f_{X}(t)=\lambda_{X} \times e^{-\lambda_{X} t}$ for ON state and $f_{Y}(t)=\lambda_{Y} \times e^{-\lambda_{Y} t}$ for OFF state. The probability that the channel $i$ will be in OFF state at time $t$, i.e., $P_{O F F}(t)$, is calculated as:

$$
P_{O F F}(t)=\frac{\lambda_{X}}{\lambda_{X}+\lambda_{Y}}+\frac{\lambda_{Y}}{\lambda_{X}+\lambda_{Y}} e^{-\left(\lambda_{X}+\lambda_{Y}\right) t}
$$

where $\lambda_{X}$ and $\lambda_{Y}$ are the rate parameter for exponential distribution.

We consider then four different PR nodes activity patterns $[5,6]$, described as follows (see Fig. 1):

- Long Term PR Activity: In Long Term PR Activity, the channel has long ON and long OFF periods. This type of $\mathrm{PR}$ activity can be seen in the scenarios where primary radio nodes subscribed to free call packages.

- High PR Activity: In High PR Activity, the channel has long ON and short OFF periods. This type of PR activity can be seen in highly congested urban environments or in rush hours, where all the channels are mostly occupied.

- Low PR Activity: In Low PR Activity, the channel has short ON and long OFF periods. This type of PR activity can be observed in remote areas or during less peak hours.

- Intermittent PR Activity: In Intermittent PR Activity, the channel has short $\mathrm{ON}$ and short OFF periods. This type of PR activity can be observed where users use the channels for very short period of time, e.g., bus stations, railway stations etc.

Fig. 1 depicts an example of these four activity patterns. In order to achieve such PR nodes activity, we vary the rate parameter $\lambda_{X}$ and $\lambda_{Y}$ of the exponential distribution, as indicated in Table $1[5,6]$. 


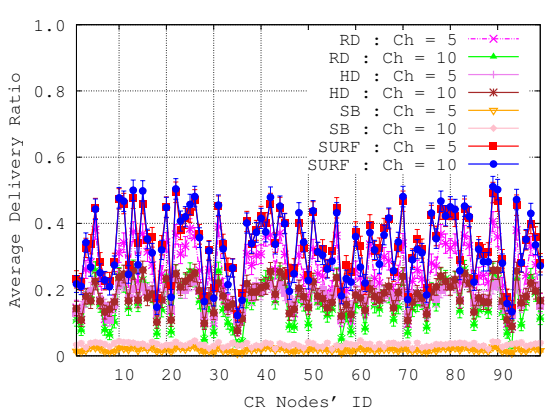

(a)

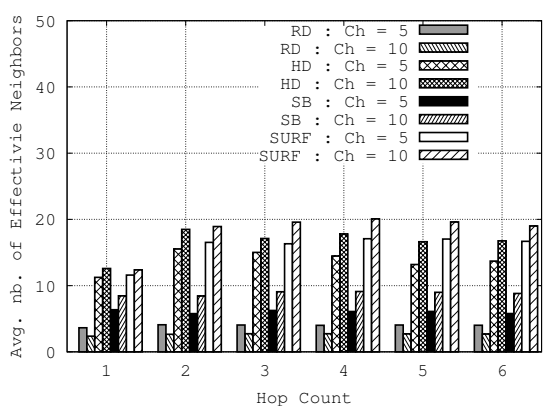

(b)

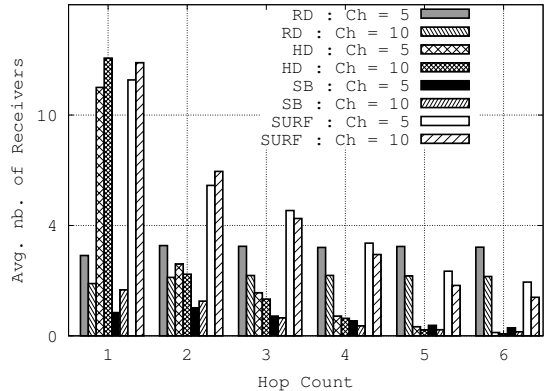

(c)

Figure 2: Zero Primary Radio Activity. (a) CR Nodes' ID and average delivery ratio for RD, HD, SB and SURF. (b) Hop count and average number of effective neighbors for RD, HD, SB and SURF. (c) Hop count and average number of receivers for RD, HD, SB and SURF.

Table 2: Harmful Interference Ratio (HIR) (in \%) under various Primary Radio Nodes Activity.

\begin{tabular}{c|cc|cc|cc|cc}
\hline & \multicolumn{3}{|c|}{$R D$} & \multicolumn{2}{c|}{$H D$} & \multicolumn{2}{c|}{$S B$} & \multicolumn{2}{c}{$S U R F$} \\
\hline & $\mathbf{C h = 5}$ & $\mathbf{C h = 1 0}$ & $\mathbf{C h}=\mathbf{5}$ & $\mathbf{C h}=\mathbf{1 0}$ & $\mathbf{C h}=\mathbf{5}$ & $\mathbf{C h}=\mathbf{1 0}$ & $\mathbf{C h = 5}$ & $\mathbf{C h = 1 0}$ \\
\hline Long Term & 63 & 53 & 51 & 49 & 50 & 50 & 23 & 27 \\
\hline High & 90 & 87 & 86 & 83 & 89 & 89 & 60 & 65 \\
\hline Low & 17 & 16 & 13 & 12 & 18 & 13 & 5 & 5 \\
\hline Intermittent & 61 & 49 & 47 & 46 & 58 & 56 & 22 & 22 \\
\hline
\end{tabular}

\section{PERFORMANCE ANALYSIS}

This section presents the performance analysis of the fours channel selection strategies under varying PR nodes activity. To achieve this, we performed extensive NS-2 simulations and considered three performance metrics:

1. Harmful Interference Ratio (HIR): This metric is defined in order to capture the notion of collision with $\mathrm{PR}$ nodes. HIR is defined as the ratio of the total number of times the channel is occupied by PR node after the channel selection decision over total number of times the channel selection decision occurs.

2. Average Delivery Ratio: This metric is defined to effectively measure the data dissemination process. It is the ratio of packets received by a particular CR node over total packets sent in the network.

3. Ratio of Accumulative CR Receivers: This metric also evaluates the data dissemination process. It is defined as the average ratio of accumulative CR receivers per hop over the accumulative effective neighbors per hop. Accumulative CR receivers per hop are the number of CR receivers per hop that successfully received the message, while accumulative effective neighbors per hop are the CR neighbors that selects the same channel for overhearing as the sender node used for transmission. Note that by accumulative ratio we mean: at each new hop $h$, the receivers and effective neighbors of all previous hops $l<h$ are summed up to the ones at hop $h$.

The number of CR nodes is fixed to 100. CRs are randomly deployed within a square area of $700 \times 700 m^{2}$ and their transmission range is set to $250 \mathrm{~m}$. Simulations run for 1000 seconds and a total of 1000 packets are sent, where each packet is sent by a randomly selected node at an interval of
1 second. All results are obtained with a confidence interval of $95 \%$.

We consider $5(C h=5)$ and $10(C h=10)$ total number of channels, which allows varying the neighborhood density $d_{\text {avg }}$ between 11.3 (when $\mathrm{Ch}=5$ ) and 20.1 (when $\mathrm{Ch}=10$ ). Note this density is computed after the spectrum sensing provides the list of available channels and before the CRs select the channel to transmit/overhear. In this case, it is worth mentioning that, at the following simulation studies, the neighborhood density varies in function of the CRs' channel selection and is lower than the above ones. The results attest the obtained low delivery ratios are mainly due to the creation of different topologies resulted from the multi-channel availability and distributed channel selection by CRs. This can be verified in the Fig. 2, which shows results for delivery ratio, number of receivers and of effective neighbors, for $\mathrm{Ch}=5$ and $\mathrm{Ch}=10$ when no $P R$ nodes activity is present in the channels. As can be observed, even when $\mathrm{CR}$ nodes do not have to compete with PR nodes to have access to the channels, the average delivery ratio ranges from $35 \%-50 \%$, the average number of effective neighbors ranges from $10-20$ and the average number of receivers ranges from $12-2$ (from $1^{\text {st }}$ to $6^{\text {th }}$ hop) in SURF.

Fig. 3-Fig. 6 show the graphs for varying PR nodes activity patterns. Similarly, Table 2 summarizes the harmful interference ratio of Fig. 3-Fig. 6. In Long Term PR activity, besides of guaranteeing lower HIR compared to RD, HD, and SB, SURF also ensures a higher delivery ratio than such approaches. In High PR activity, all the channel are highly occupied, and consequently, very less chances for communication is let to all the approaches. Nevertheless, SURF is able to manage very low HIR and still have some delivery ratio ( $2 \%$ around), compared to the other approaches.

It is clear that when PR activity is very low (cf Fig. 5) every strategy behaves well in term of HIR (cf. 5(a)). In this case, SURF helps select the best channel in term of CR connectivity, i.e., delivery ratio to CR (cf. Fig. 5(b)), while 


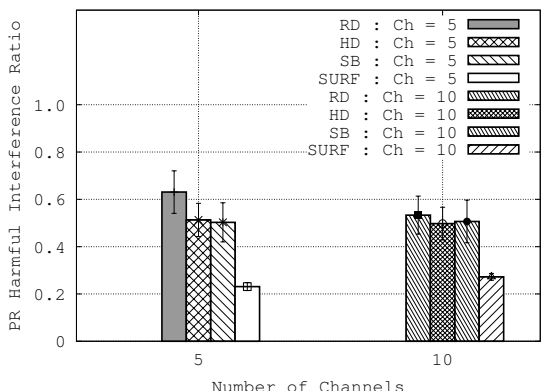

(a)

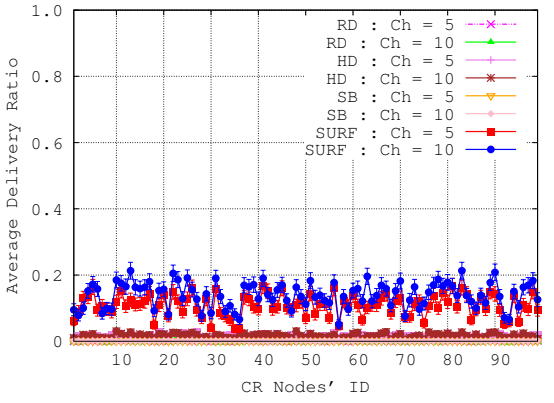

(b)

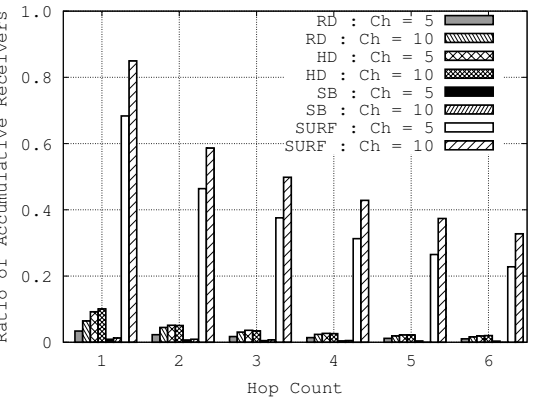

(c)

Figure 3: Long Term Primary Radio Activity. (a) PR harmful interference ratio for RD, HD, SB and SURF. (b) CR Nodes' ID and average delivery ratio for RD, HD, SB and SURF. (c) Hop count and Ratio of accumulative receivers for RD, HD, SB and SURF.

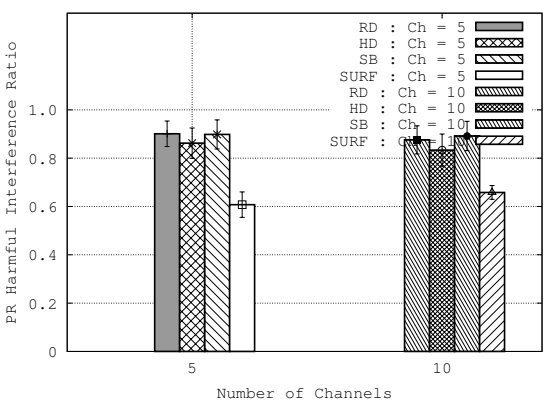

(a)

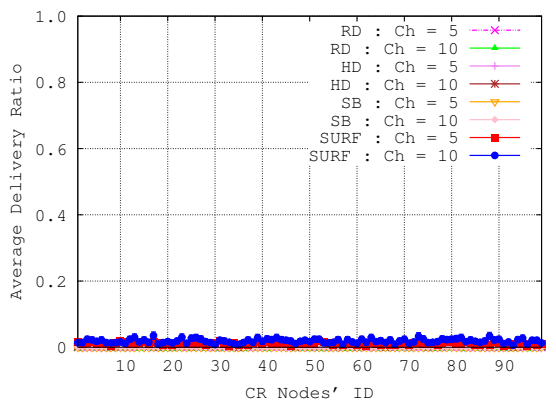

(b)

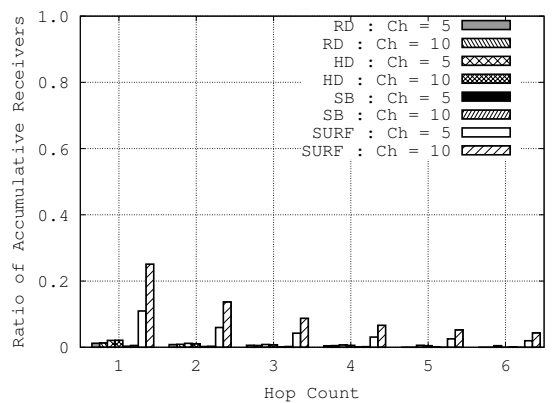

(c)

Figure 4: High Primary Radio Activity. (a) PR harmful interference ratio for RD, HD, SB and SURF. (b) CR Nodes' ID and average delivery ratio for RD, HD, SB and SURF. (c) Hop count and Ratio of accumulative receivers for RD, HD, SB and SURF.

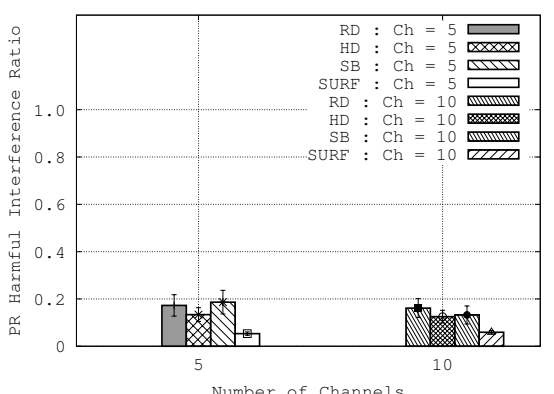

(a)

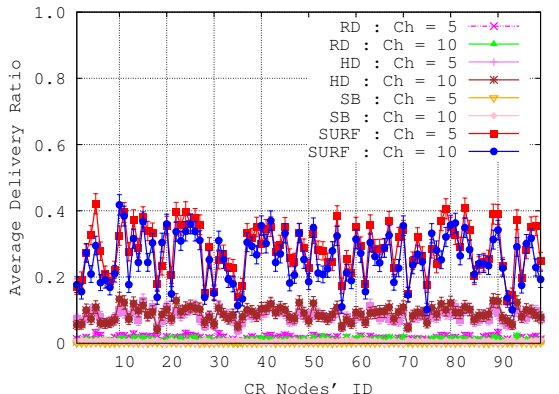

(b)

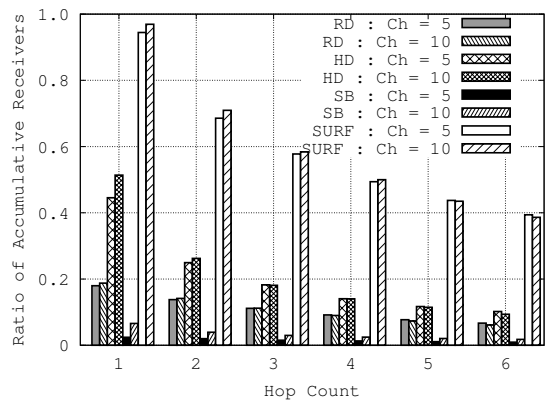

(c)

Figure 5: Low Primary Radio Activity. (a) PR harmful interference ratio for RD, HD, SB and SURF. (b) CR Nodes' ID and average delivery ratio for RD, HD, SB and SURF. (c) Hop count and Ratio of accumulative receivers for RD, HD, SB and SURF.

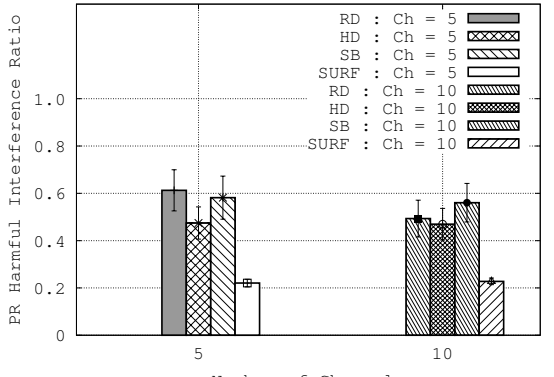

(a)

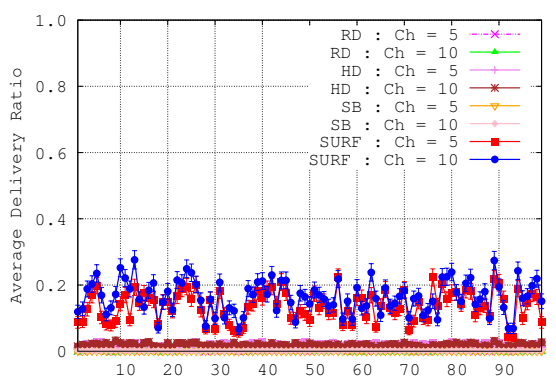

(b)

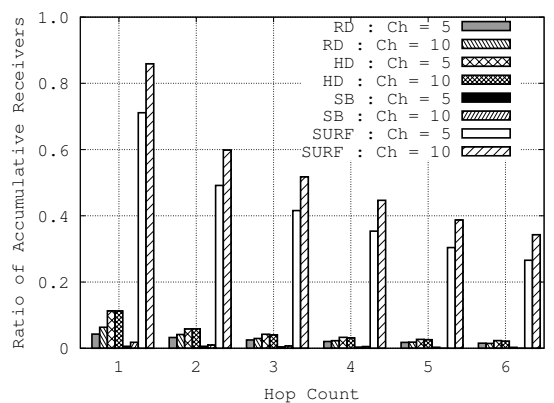

(c)

Figure 6: Intermittent Primary Radio Activity. (a) PR harmful interference ratio for RD, HD, SB and SURF. (b) CR Nodes' ID and average delivery ratio for RD, HD, SB and SURF. (c) Hop count and Ratio of accumulative receivers for RD, HD, SB and SURF. 
generates very less or almost zero HIR, when compared to $\mathrm{RD}, \mathrm{SB}$, and $\mathrm{HD}$. The receivers ratio is also the highest for SURF.

Unsurprisingly, the best performance gain is observed in the intermittent case when using SURF: Lower HIR and higher delivery ratio is provided than $\mathrm{RD}, \mathrm{HD}$, and $\mathrm{SB}$. It is worth noting that, in the cases where short $\mathrm{ON}$ for $\mathrm{PR}$ nodes is considered (i.e., in intermittent or low activity scenarios), all the approaches perform the better. However, the channel selection mechanism provided by SURF could find the best spectrum opportunities in all considered cases, while respecting the PR nodes activities.

Main Conclusions. The main conclusions are:

- When the system is free (Low PR activity), every solution offers acceptable gain. Sometimes a clever solution is not worth it due to the complexity it introduces.

- When the system is close to maximum capacity (High PR activity), all solutions have bad performance. When channels are fully occupied by PRs there is no real opportunity for transmission, here also the gain is very low compared to the complexity of the solutions.

- Intermittent case is the case where clever solutions need to operate. This is where SURF gives the best results and the target region to avail communication opportunities.

\section{IMPROVEMENTS REGARDING SURF}

The channel selection strategy provided by SURF can be further enhanced by considering the primary radio nodes activity pattern. In the previous section, we have pointed out that the intermittent case is the case where clever solutions need to operate. In this regard, we can evaluate the "power" of using other history-based metrics (that try to better infer the quality of channels) combined with the current $P_{w}$ of SURF. SURF is then required to keep track of history of past PR nodes activity. This history could be used to give more weight to the channels with short $\mathrm{ON}$ in average. Some examples of metrics are given below:

1) How often the channel is free? Here, SURF may keep history of channel ON/OFF states. SURF will consider an "observation time window". The observation time window is defined as the duration of time during which the channel ON/OFF states are observed. In this manner, SURF will compute the ratio of being free over the time window (the size of the time window could be varied to evaluate the impact of ON/OFF states).

2) How long channels stay in OFF state? Here, SURF may compute the duration of OFF state over the total time of window size, in the considered time window. This metric depends on how SURF keeps the history of channel states. This could be done on per time slot basis or combining tow or more tim slots.

3) What was the ratio of success (reception or transmission) over the times the channel was in OFF state? This metric will give the quality of the channel in terms of contention. By using this metric, SURF may avoid those channels that are quality wise poor.

Note that all the aforementioned three metrics depend upon when the verification for a free channel is performed (periodically or only when a packet event reception or transmission happens.)

\section{CONCLUSION AND FUTURE WORK}

In this paper, we studied the impact of primary radio nodes activity on four channel selection strategies i.e. RD, HD, SB, and SURF. To achieve this, we performed extensive NS-2 simulations. We observed that the channel selection strategies are greatly influenced by the primary radio nodes activity. More particularly, our channel selection strategy SURF outperformed RD, HD, and SB in terms of delivery ratio and causes less harmful interference to PR nodes, in all primary radio nodes activity pattern. As plan of our future work, we intend to improve SURF by considering the metrics discussed in section 5 .

\section{REFERENCES}

[1] I. F. Akyildiz, W.-Y. Lee, M. C. Vuran, and S. Mohanty, "Next generation/dynamic spectrum access/cognitive radio wireless networks: a survey," Computer Networks: The International Journal of Computer and Telecommunications Networking, vol. 50 , Issue 13, pp. $2127-2159,2006$.

[2] W.-Y. Lee and I. Akyildiz, "Optimal spectrum sensing framework for cognitive radio networks," IEEE Transactions on Wireless Communications, vol. 7 , no. 10, pp. $3845-3857$, october 2008.

[3] G. Yuan, R. Grammenos, Y. Yang, and W. Wang, "Performance analysis of selective opportunistic spectrum access with traffic prediction," IEEE Transactions on Vehicular Technology, vol. 59, no. 4, pp. 1949 -1959, may 2010 .

[4] A. W. Min and K. G. Shin, "Exploiting multi-channel diversity in spectrum-agile networks," in Proceedings of INFOCOM, 13-18 April 2008, pp. $1921-1929$.

[5] M. Di Felice, K. R. Chowdhury, and L. Bononi, "Modeling and performance evaluation of transmission control protocol over cognitive radio ad hoc networks," in Proceedings of the 12th ACM international conference on Modeling, analysis and simulation of wireless and mobile systems, ser. MSWiM '09. New York, NY, USA: ACM, 2009, pp. 4-12. [Online]. Available: http://doi .acm.org/10.1145/1641804.1641809

[6] S. Bayhan and F. Alagöz, "Distributed channel selection in crahns: A non-selfish scheme for mitigating spectrum fragmentation," Ad Hoc Networks, vol. In Press, Corrected Proof, pp. -, 2011. [Online]. Available: http://www. sciencedirect.com/science/article/pii/S1570870511000941

[7] J. Riihijarvi, J. Nasreddine, and P. Mahonen, "Impact of primary user activity patterns on spatial spectrum reuse opportunities," in European Wireless Conference (EW 2010), 2010.

[8] M. H. Rehmani, A. C. Viana, H. Khalife, and S. Fdida, "Improving data dissemination in multi-hop cognitive radio ad-hoc networks," in 3rd International ICST Conference on Ad Hoc Networks (ADHOCNETS 2011), Paris, France - (To appear)., 21-23 Sep 2011.

[9] Y. R. Kondareddy and P. Agrawal, "Selective broadcasting in multi-hop cognitive radio networks," in IEEE Sarnoff Symposium, Princeton, New Jersy, 28-30 April 2008, pp. 1-5.

[10] M. H. Rehmani, A. C. Viana, H. Khalife, and S. Fdida, "Surf: A distributed channel selection strategy for data dissemination in multi-hop cognitive radio networks," INRIA, Tech. Rep., 2011. [Online]. Available: http://hal.inria.fr/inria-00596224/en/

[11] B. Vujicic, N. Cackov, S. Vujicic, and L. Trajkovic, "Modeling and characterization of traffic in public safety wireless networks," in In Proc. of SPECTS, 2005, pp. 214-223.

[12] [Online]. Available: http://www.fcc.gov/pshs/public-safety-spectrum/700-MHz/

[13] S. Geirhofer, L. Tong, and B. M. Sadler, "Dynamic spectrum access in wlan channels: Emperical model and its stochastic analysis," in $A C M$ TAPAS, Aug 2006.

[14] A. Adas, "Traffic models in broadband networks," IEEE Communications Magazine, vol. 35, no. 7, pp. 82 -89, jul 1997

[15] H. Kim and K. Shin, "Efficient discovery of spectrum opportunities with mac-layer sensing in cognitive radio networks," IEEE Transactions on Mobile Computing, vol. 7, no. 5 , pp. $533-545$, may 2008 